\title{
Self-Titration by Experienced E-Cigarette Users: Blood Nicotine Delivery and Subjective Effects
}

Lynne E Dawkins ${ }^{1} \mathrm{PhD}^{*}$, Catherine F Kimber ${ }^{2} \mathrm{MBPsS}$, Mira Doig ${ }^{3} \mathrm{PhD}$, Colin Feyerabend ${ }^{3}$ $\mathrm{PhD}$, Olivia Corcoran ${ }^{4} \mathrm{PhD}$

${ }^{1}$ Division of Psychology, School of Applied Sciences, 103 Borough Road, London South Bank University, London, SE1 0AA, UK

2 Drugs and Addictive Behaviours Research Group, School of Psychology, University of East London, Water lane, E15 4LZ, UK

${ }^{3}$ ABS Laboratories Ltd., Biopark, Broadwater Road, Welwyn Garden City, Hertfordshire AL7 $3 \mathrm{AX}, \mathrm{UK}$

${ }^{4}$ Medicines Research Group, School of Health, Sport and Bioscience, University of East London, Water lane, E15 4LZ, UK

Corresponding author*: Associate Professor of Psychology, Division of Psychology, School of Applied Sciences, Room E339; 103 Borough Road, London South Bank University, London, SE1 0AA, UK

T: $+44(0) 2078155422$

Email: dawkinl3@1sbu.ac.uk

Funding: This study was fully funded by the University of East London PhD Excellence Studentship to CK.

Declaration of interests: We declare that we have received no support from any companies for this work and there are no non-financial conflicts of interest that would be considered relevant to this work. LED has previously conducted research for several independent electronic cigarette companies. These companies had no input into the design, conduct or write up of the projects. She has also acted as a consultant for the pharmaceutical industry and as an expert witness in a patent infringement case. $\mathrm{CK}$ and $\mathrm{OC}$ declare no competing interests. MD and CF have previously supported other research groups by the quantification of cotinine, 3-hydroxy cotinine and nicotine. These groups had no input into the design, conduct or write up of this project.

Keywords: Nicotine, Titration, E-cigarette, Puffing topography, Compensation 


\begin{abstract}
Rationale Self-titration is well documented in the tobacco literature. The extent to which ecigarette users (vapers) self-titrate is unknown.
\end{abstract}

Objective This study explored the effects of high and low nicotine strength liquid on puffing topography, nicotine delivery and subjective effects in experienced vapers.

Methods Eleven experienced male vapers completed 60 minutes of ad libitum vaping under low $(6 \mathrm{mg} / \mathrm{mL})$ and high $(24 \mathrm{mg} / \mathrm{mL})$ nicotine liquid conditions in two separate sessions. Measurements included: puffing topography (puff number, puff duration, volume of liquid consumed); and changes in: plasma nicotine levels, craving, withdrawal symptoms, selfreported hit, satisfaction and adverse effects.

Results Liquid consumption and puff number were higher, and puff duration longer, in the low nicotine strength condition (all ps $<0.01$ ). The mean difference in nicotine boost from baseline in the low condition was $8.59(7.52) \mathrm{ng} / \mathrm{mL}, 16.99(11.72) \mathrm{ng} / \mathrm{mL}$ and $22.03(16.19) \mathrm{ng} / \mathrm{mL}$ at 10, 30 and 60 minutes respectively. Corresponding values for the high condition were 33.77 (34.88) ng/mL, 35.48 (28.31) ng/mL and 43.57 (34.78) ng/mL (ps < 0.05). There were no statistically significant differences between conditions in self-reported craving, withdrawal symptoms, satisfaction, hit or adverse effects.

Conclusions Vapers engaged in compensatory puffing with lower nicotine strength liquid, doubling their consumption. Whilst compensatory puffing was sufficient to reduce craving and withdrawal discomfort, self-titration was incomplete with significantly higher plasma nicotine levels in the high condition. 


\section{Introduction}

Electronic cigarettes (e-cigarettes) are battery-operated devices which deliver nicotine via an aerosol typically containing nicotine (from $0 \mathrm{mg} / \mathrm{mL}$ concentration to $36 \mathrm{mg} / \mathrm{mL}$ ), propylene glycol, vegetable glycerine and flavourings. E-cigarette use is on the increase: from 2.1 million users in 2014 to 2.6 million in May 2015 in Great Britain, mostly comprising ex-smokers (ASH, 2015). Their increasing popularity and efficacy for quitting over conventional Nicotine Replacement Therapy (NRT) (Brown, Beard, Kotz, Michie \& West, 2014) is commonly attributed to the ability to mimic the behavioural actions of cigarette smoking and to provide an analogous method of nicotine delivery (Polosa, et al., 2014). Although early studies in naive users reported poor nicotine delivery with little to no increase in blood nicotine levels (Bullen, McRobbie, Thornley, Glover, Lin \& Laugesen), 2010; Eissenberg, 2010), later studies in experienced users and with newer models (Farsalinos, Spyrou, Tsimopoulou, Stefopoulos, Romagna \& Voudris, 2014) have found pronounced increases paralleled by significant cigarette craving reduction (Etter, 2015). In fact, direct comparisons have demonstrated a 50\% higher blood nicotine level in experienced versus naive e-cigarette users (Farsalinos et al., 2015) and with a newer versus an older generation device (Farsalinos et al., 2014).

Compared with smokers using tobacco cigarettes or e-cigarettes, experienced e-cigarette users (vapers) typically take longer puffs (Lee, Gawron \& Goniewicz, 2015), although smokers may adjust their puffing patterns within a week of switching to e-cigarettes (Hua, Yip \& Talbot, 2013; Lee, Gawron \& Goniewicz, 2015; Talih et al., 2014). Machine-yield data consistently show that compared to tobacco cigarettes, e-cigarettes require a stronger suction to produce the aerosol (Trtchounian, Williams \& Talbot, 2010). Thus longer e-cigarette puffs may be required to compensate for the less effective delivery method compared to tobacco smoking.

Blood nicotine levels may also be influenced by puffing patterns (such as puff length, volume and frequency, known as puffing topography). We have previously observed large individual differences in plasma nicotine levels ranging from $13.4 \mathrm{ng} / \mathrm{mL}$ to $2.50 \mathrm{ng} / \mathrm{mL}$ after ten e-cigarette puffs, suggesting that users adjust their puff duration and/or volume to maintain a desired nicotine level (i.e. self-titrate) (Dawkins \& Corcoran, 2014). More recently, Ramôa and colleagues (Ramôa et al., 2015), using a ten-puff protocol reported that e-cigarette users increased their puff duration with $0 \mathrm{mg} / \mathrm{mL}$ compared with $36 \mathrm{mg} / \mathrm{mL}$ nicotine concentration (but not 8 or $18 \mathrm{vs} .36 \mathrm{mg} / \mathrm{mL}$ ) which may reflect an attempt to self-titrate due to the absence of nicotine (Ramôa et al., 2015). Although this provides some evidence of titration, the use of a standardised ten min ten-puff protocol restricts users' ability to compensate fully. Recent pilot work in our laboratory using an ad libitum design with low (6 
$\mathrm{mg} / \mathrm{mL})$ and high $(18 \mathrm{mg} / \mathrm{mL})$ nicotine strength liquids also evidences compensatory puffing behaviour (greater liquid consumption and puff number, and longer puff duration in the low condition) and was the basis for this study. Taken together these studies are suggestive of compensatory puffing behaviour in e-cigarette users.

Compensatory smoking or nicotine titration is well documented in the tobacco smoking population; when given lower nicotine cigarettes, smokers increase their inhalation by taking more frequent and longer puffs or consuming more cigarettes (Ashton, 1979; Ashton \& Watson, 1970; Russell, 1980; Russell, Jarvis, Iyer \& Feyerabend, 1980; Russell, Wilson, Patel, Feyerabend \& Cole, 1975; Woodward \& Tunstall-Pedoe, 1993). However, whilst self-report data (e.g. satisfaction) suggests that compensation could be effective (Sutton, Russell, Iyer, Feyerabend, \& Saloojee, 1982), biomarkers suggest that compensation is seldom complete (Russell, Sutton, Iyer, Feyerabend, \& Vesey, 1982). Conversely, there is no such direct empirical evidence from ad libitum protocols in e-cigarette users. Many e-cigarettes users gradually reduce their nicotine concentration over time (Polosa, Caponnetto, Cibella \& Le-Houezec, 2015). Others may be obliged to do so with the implementation of the Tobacco Product Directive EU-TPD in Europe in May 2016, which will ban products exceeding $20 \mathrm{mg} / \mathrm{mL}$ nicotine concentrations (unless licensed as a medicine, for example, in the UK). Some, however, may need to increase their nicotine concentration in order to achieve complete smoking abstinence in the early stages of a cessation attempt (Farsalinos, Romagna, Tsiapras, Kyrzopoulos \& Voudris, 2013). Documenting the behavioural and pharmacokinetic effects of switching to a lower nicotine strength liquid are therefore expedient in the current regulatory environment.

We aimed to investigate to what extent, if at all, e-cigarette users self-titrate when given a lower nicotine strength liquid. We hypothesised that users will attempt to compensate by consuming more liquid and taking longer and more frequent puffs when using a lower nicotine strength liquid. Secondary aims were to explore subjective effects (craving, withdrawal symptoms, positive and negative reactions) and plasma nicotine concentrations between conditions (high vs. low nicotine concentration liquid).

\section{Methods}

\section{Design and ethical approval}

The study received full ethical approval from the University of East London's ethics committee (UREC_1415_40) and was conducted in accordance with the ethical standards outlined in the 1964 
Declaration of Helsinki. All participants provided written informed consent to take part in the study and for the study to be written up for publication.

A double-blind within-participants, counterbalanced design with 2 conditions: 'low' $(6 \mathrm{mg} / \mathrm{mL})$ and 'high' $(24 \mathrm{mg} / \mathrm{mL})$ nicotine concentration liquid was used. Eleven (initially 12 but one was removed from all analyses due to inability to provide blood) regular e-cigarette users (all male ex-smokers), attended 2 separate sessions between 2 and 7 days apart at the University of East London. Participants responded to advertisements on social media sites, e-cigarette forums and emails. Eligibility criteria were: aged 18+; male (for ease of providing blood samples, see Dawkins \& Corcoran, 2014); experienced e-cigarette user (daily use for $>3$ months); currently using a second or third generation e-cigarette; familiar with $24 \mathrm{mg} / \mathrm{mL}$ nicotine concentration liquid (i.e. used $24 \mathrm{mg} / \mathrm{mL}$ at least once in the last 6 months); baseline salivary cotinine levels $>100 \mathrm{ng} / \mathrm{mL}$; CO levels $\leq 10 \mathrm{ppm}$; willing to provide saliva and blood samples and abstain from using nicotine for $12 \mathrm{~h}$ prior to study commencement.

\section{Randomisation and Masking}

Ten most popular brands of nicotine liquid were selected (search conducted in January/ February 2015) and purchased online. One member of the research team selected a brand (Halo Smokers' Angels) at random with nicotine concentrations of 6 and $24 \mathrm{mg} / \mathrm{mL}$ (50/50 ratio of propylene glycol to glycerine). Liquid was decanted into plain bottles and re-labelled X and Y, so brand and nicotine concentration were unknown to participants and participant-facing researchers. One researcher was responsible for filling the $\mathrm{eVic}^{\mathrm{TM}}$, another collected questionnaire data, and a phlebotomist collected blood; all were blind to nicotine condition. Order of presentation was counterbalanced and participants were randomly allocated to receive either X or Y first.

\section{Procedures}

Prior to testing, participants were sent an information sheet and screened via email and telephone. Pre-test saliva samples were collected by post and assessed for cotinine at Advanced Bioanalytical Service (ABS) Laboratories Ltd. An 'eVic ${ }^{\mathrm{TM}}$ supreme' e-cigarette from Joyetech, fitted with a 'Nautilus Aspire' tank was set at $3.9 \mathrm{~V}(8.5 \mathrm{~W})$ for an atomiser resistance of $1.8 \mathrm{ohm}$, and adjusted to the largest airflow. The tank was weighed at the beginning and end of each session to calculate the weight of liquid consumed. This was then divided by the density of the liquid to calculate $\mathrm{mL}$ consumed for each participant in each of the two conditions. 
Participants abstained from e-cigarette and nicotine use for $12 \mathrm{~h}$ prior to study commencement and were tested individually. Upon arrival, participants were offered a glass of water and provided written informed consent. Smoking status was verified via carbon monoxide breath test and nicotine abstinence via baseline blood sample.

Baseline and demographic characteristics including vaping history, e-cigarette dependence (measured via an adapted version of the Fagerström Test of Nicotine Dependence (Fagerström, 2012; eFTND, as described by Farsalinos et al., 2014), single-item rating of addiction from $0-100 \%$ (scored from 0 $=$ low addiction to $5=$ very high addiction taken from the Cigarette Dependence Scale (CDS; Etter, Le Houezec \& Perneger, 2003), current craving ('urge to vape') and withdrawal symptoms (Mood and Physical Symptoms Scale; MPSS) (West \& Hajek, 2004) were collected before a phlebotomist inserted a venous cannula into the participant's forearm and collected a baseline blood sample. Thereafter, the participant was presented with the $\mathrm{eVic}^{\mathrm{TM}}$ e-cigarette and asked to vape ad libitum for $60 \mathrm{~min}$. During this time participants read quietly, worked or engaged with social/other media using their own devices. Further blood samples were taken, and craving and withdrawal symptoms recorded, at 10, 30 and 60 min after the first puff. Puffing topography (puff number and puff duration) was recorded by the $\mathrm{eVic}^{\mathrm{TM}}$ and downloaded to 'My Vapors Joyetech 1.4'. Puff number and duration recorded using the $\mathrm{eVic}^{\mathrm{TM}}$ correlated highly with participant observation in our pilot study $(\mathrm{r}=.993$, $\mathrm{p}<.001 ; \mathrm{r}=.924, \mathrm{p}=.025$ for puff numbers and duration under low nicotine concentration respectively). The venous catheter was removed following the last blood sample collection at $60 \mathrm{~min}$, before completion of a Visual Analogue Scale assessing positive (hit and satisfaction) and adverse effects associated with nicotine and e-cigarette use (Dawkins \& Corcoran, 2014). Participant were then offered a snack and refreshment, thanked and reimbursed financially for their time (£50 compensation) at the end of the second session.

\section{Blood collection and nicotine analysis}

Blood samples were collected using 4mL BD K2EDTA vacutainer tubes and stored in a polystyrene igloo away from the vaping area. Only the phlebotomist and a designated researcher (Hepatitis B vaccinated) handled blood samples. Prior to handling vacutainer tubes, hands were disinfected (with Virucidal alcohol hand gel) and gloves were worn and changed each time to avoid contamination. A different researcher handled the $\mathrm{eVic}^{\mathrm{TM}}$ and liquid to avoid blood sample contamination. After each testing session all blood samples were transported to an on-site forensic laboratory for plasma extraction. 
Vacutainer tubes were placed in a 'MSE Falcon 6/300R' centrifuge system set to run as follows: 2000 $\mathrm{RCF}$, Temperature $4^{\circ} \mathrm{C}$, for $10 \mathrm{~min}$, to render samples acellular and obtain plasma. All samples were kept at $-20{ }^{\circ} \mathrm{C}$ pending transportation to ABS Laboratories Ltd. for analysis using a validated LCMS/MS method with a lower limit of quantification (LOQ) of $0.5 \mathrm{ng} / \mathrm{mL}$.

\section{Outcomes}

The primary outcome was puffing topography: i) mean number of puffs; ii) mean puff duration and, iii) volume of liquid consumed (in millilitres). Secondary outcomes were: change in plasma nicotine levels, change in craving and withdrawal symptoms and, self-reported hit, satisfaction and adverse effects. Change in plasma nicotine level ('nicotine boost') was calculated by subtracting baseline level from levels at 10, 30 and $60 \mathrm{~min}$ and reduction in craving and withdrawal symptoms by subtracting scores at 10, 30 and 60 from baseline scores.

\section{Statistical analysis}

Required sample size for detecting an effect on puffing topography was calculated using GPower 3.1 using our pilot data. To detect a difference between conditions in mean number of puffs, puff duration and volume consumed at $\mathrm{p}<0.05$ with $95 \% \mathrm{CI}$, a sample of between $\mathrm{N}=9$ and 17 was required. We planned to recruit 15 participants but due to participant drop out and failure to meet required baseline criteria, only 11 were tested.

Data were analysed using 'IBM SPSS Statistics 22'. Non-parametric statistics (Wilcoxon signed-rank tests for paired samples) were conducted to compare data between the high and low nicotine conditions as variable data were not normally distributed and transformations failed to correct to normal. Spearman correlations were used to explore the relationship between plasma nicotine and puffing topography data. Accepted $\alpha$ level was 0.05 (all 2-tailed tests).

\section{Results}

\section{Participant Characteristics}

Table 1 provides demographics baseline characteristics of the sample 
Table 1 Participants' demographics and Baseline characteristics

Gender

Male

Female

Age (years)

Ethnicity

White

Qualification

GSCEs level

A levels

Undergraduate level (5 to 6 )

Occupational status

Employed

Non-employed

Self-employed

eFTND $^{1}$

E-cig Addiction ${ }^{2}$

Baseline cotinine levels (ng/mL)

3-hydroxy-cotinine levels $(\mathrm{ng} / \mathrm{mL})$

Cotinine to 3-hydroxy-cotinine ratio

Baseline CO levels high strength

(ppm)

Baseline CO levels low strength (ppm)

\section{N Percent.}

$11 \quad 100$

$0 \quad 0$

11

$11 \quad 100$

SD

Min

$\operatorname{Max}$

Mean

SD

(Min 


\section{Puffing Topography}

We observed some instances of extremely short (i.e. $<1$ s) puffs/button presses $(9 / 568$ puffs in the high condition and $15 / 887$ puffs in the low condition; $1.58 \%$ and $1.69 \%$ respectively). These were verified against backup video data and deleted in cases where the device was not clearly in the mouth $(\mathrm{N}=4)$. In all other instances the short puff preceded or succeeded a longer puff (by between 0.1 and $1 \mathrm{~s})$ and were therefore merged with the longer puff.

There was a statistically significant difference between conditions in mean puff number, duration and volume of liquid $(\mathrm{mL})$ consumed providing clear evidence for compensatory puffing (see Table 2).

Table 2 Puffing topography (mean number of puffs and mean duration)

High $\quad$ Low $\quad$ Z

$\begin{array}{lcccc}\text { Puff number } & 48.36(22.86) & 70.73(34.45) & -2.40 & 0.016 \\ & & & & \\ \text { Puff duration (s) } & 3.84(1.02) & 5.20(1.39) & -2.93 & 0.003 \\ \begin{array}{l}\text { Liquid consumed } \\ \text { (mL) }\end{array} & 0.64(0.19) & 1.23(0.59) & -2.93 & 0.003\end{array}$

\section{Blood Nicotine Delivery}

Figure 1 displays mean nicotine plasma levels over time for the low $(6 \mathrm{mg} / \mathrm{mL})$ and high $(24 \mathrm{mg} / \mathrm{mL})$ nicotine liquid conditions. The mean (SD) difference in nicotine boost from baseline for the low nicotine condition was 8.59 (7.52) ng/mL, 16.99 (11.72) ng/mL and 22.03 (16.19) $\mathrm{ng} / \mathrm{mL}$ at 10, 30 and 60 minutes respectively. Corresponding values for the high nicotine condition were 33.77 (34.88) $\mathrm{ng} / \mathrm{mL}, 35.48$ (28.31) $\mathrm{ng} / \mathrm{mL}$ and 43.57 (34.78) $\mathrm{ng} / \mathrm{mL}$. These differences in plasma nicotine levels between conditions were statistically significant at each time point $(10 \mathrm{~min}: Z=-2.85, p=0.04 ; 30$ $\min : Z=-2.70, p=0.007 ; 60 \min : Z=-2.58, p=0.01)$. 


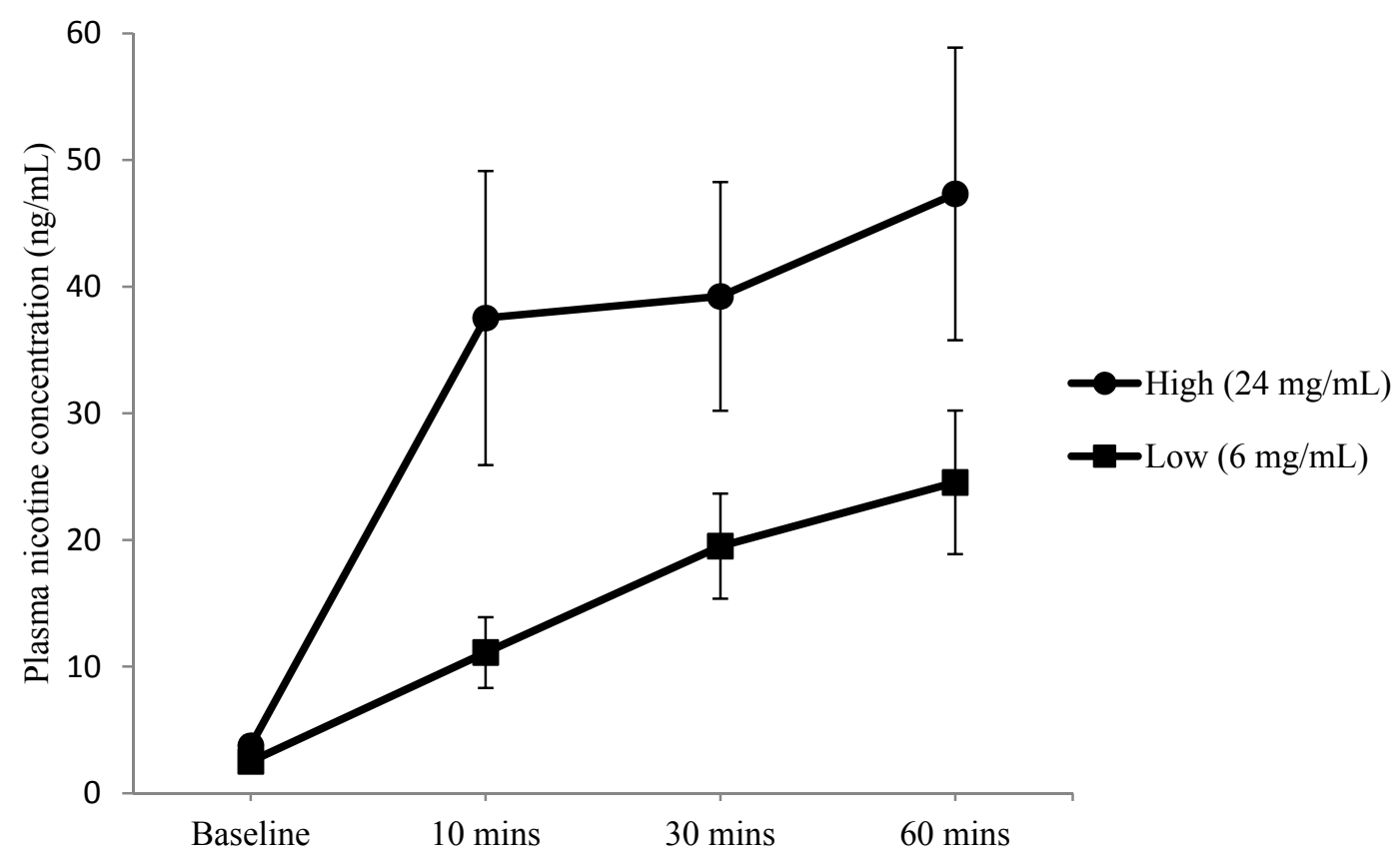

Fig 1 Plasma nicotine concentration at baseline, 10, 30 and 60 min from the first puff, for the high and low nicotine conditions $(N=11$; Error bars are \pm 1 SEM)

Correlations between puffing topography indices and plasma nicotine levels

Puffing topography variables correlated positively with nicotine boost at each time point under both the high and low conditions (see Table 3). Effects were generally stronger in the high condition and for volume of liquid consumed and puff number; results did not reach statistical significance in the low condition for puff duration.

Table 3 Correlations between plasma nicotine boost at each time point and puff number, puff duration and liquid consumption in the high and low nicotine conditions.

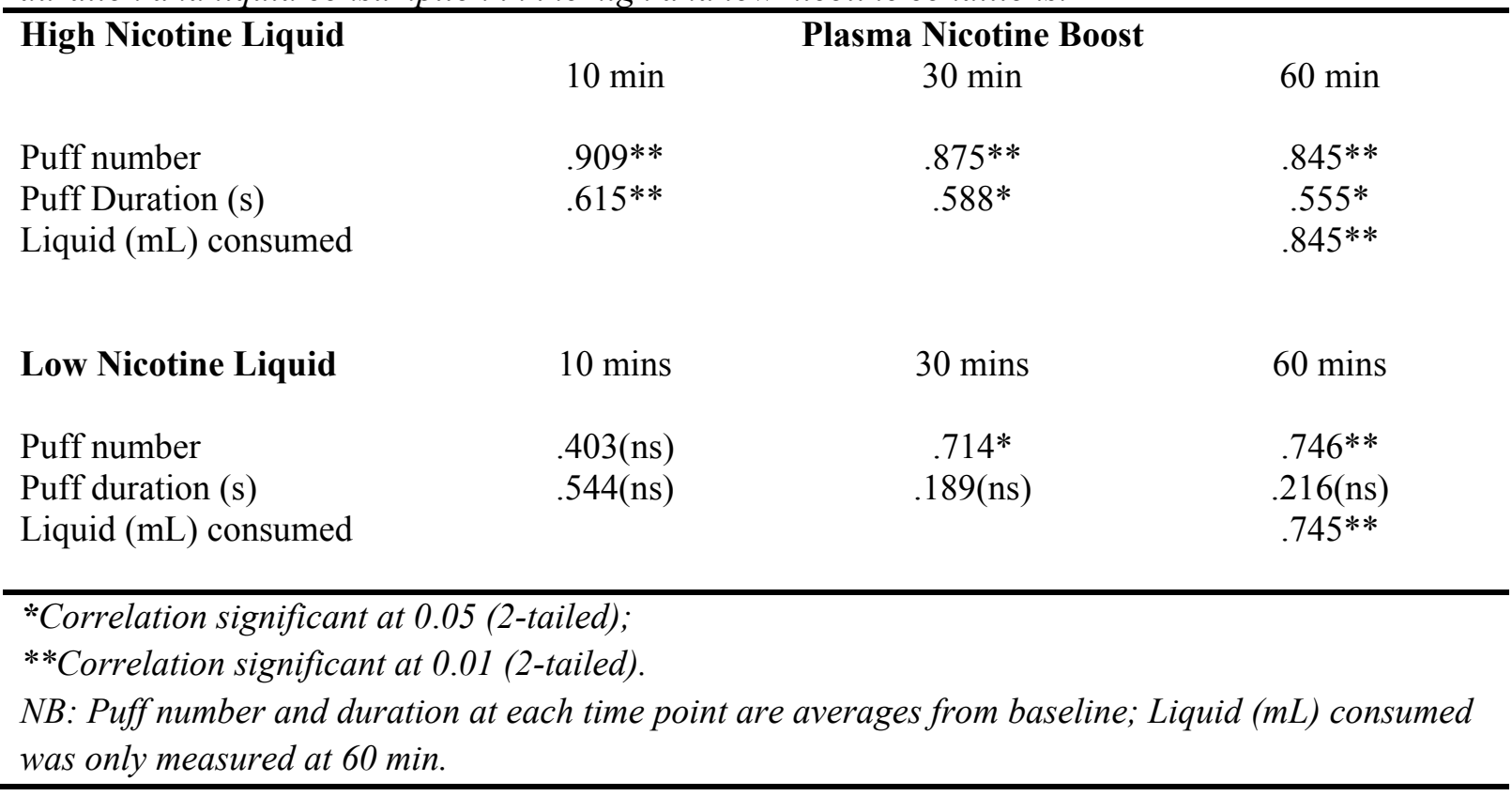


Neither baseline cotinine, 3-hydroxy-cotinine or cotinine to 3-hydroxy cotinine ratio correlated with blood nicotine boost under high or low conditions at any time point (all $r s<0.44$; all $p s>0.20$ ) or with puffing topography variables (all $r s<0.05$, all $p s>0.12$ ), indicating that puffing topography was not affected by participants' previous nicotine use or phenotypic status to metabolise nicotine.

\section{Subjective Effects}

As can be seen in Figures 2 and 3, self-reported urge to vape and nicotine-related withdrawal symptoms decreased over time in both the high and low nicotine liquid conditions. Change scores from baseline did not differ between conditions at any time point (all $\mathrm{Z}$ scores $<1, p>0.3$ ).

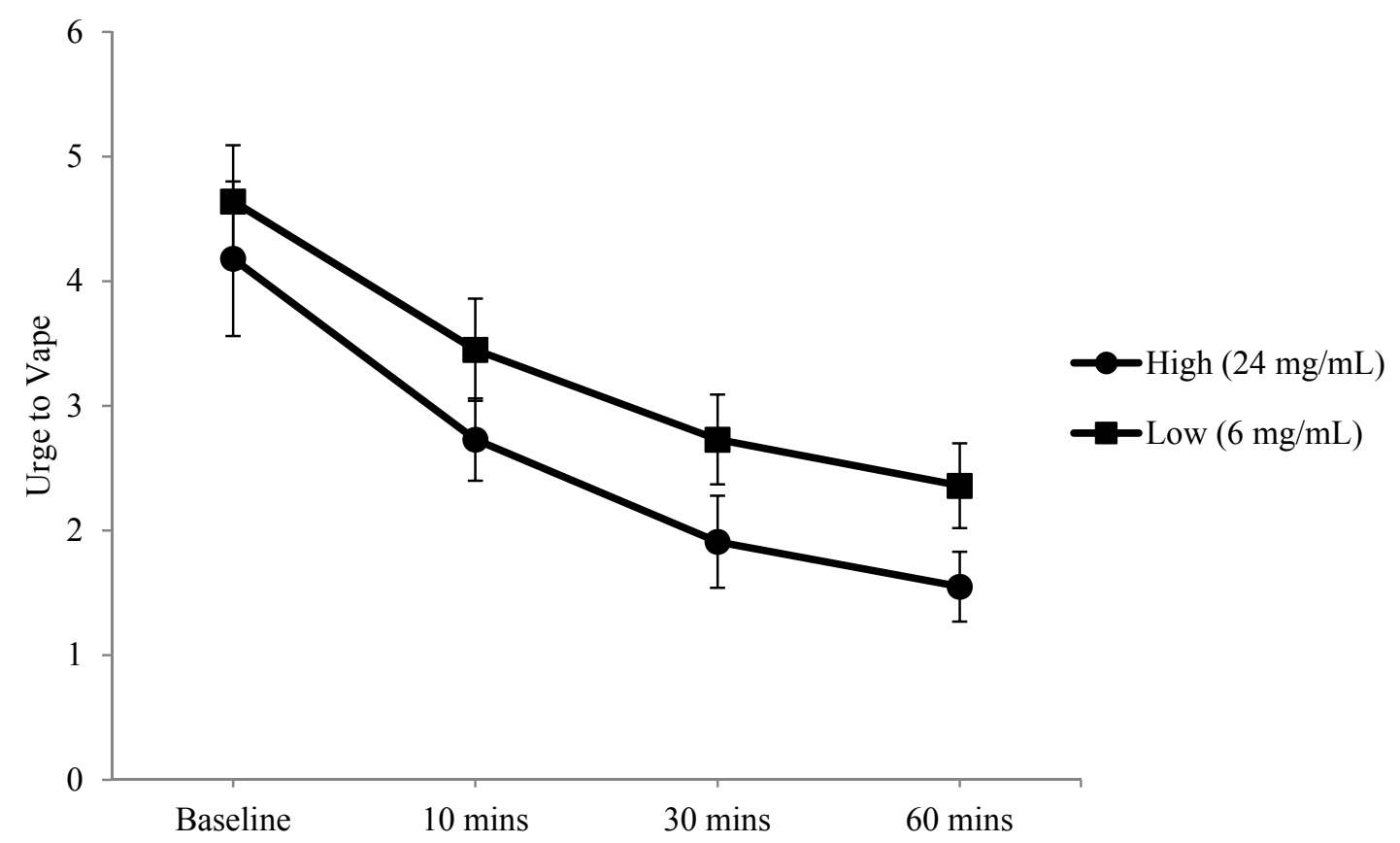

Fig. 2 Urge to vape at baseline, 10,30 and 60 min for the high and low nicotine conditions $(N=11$; Error bars are \pm 1 SEM) 


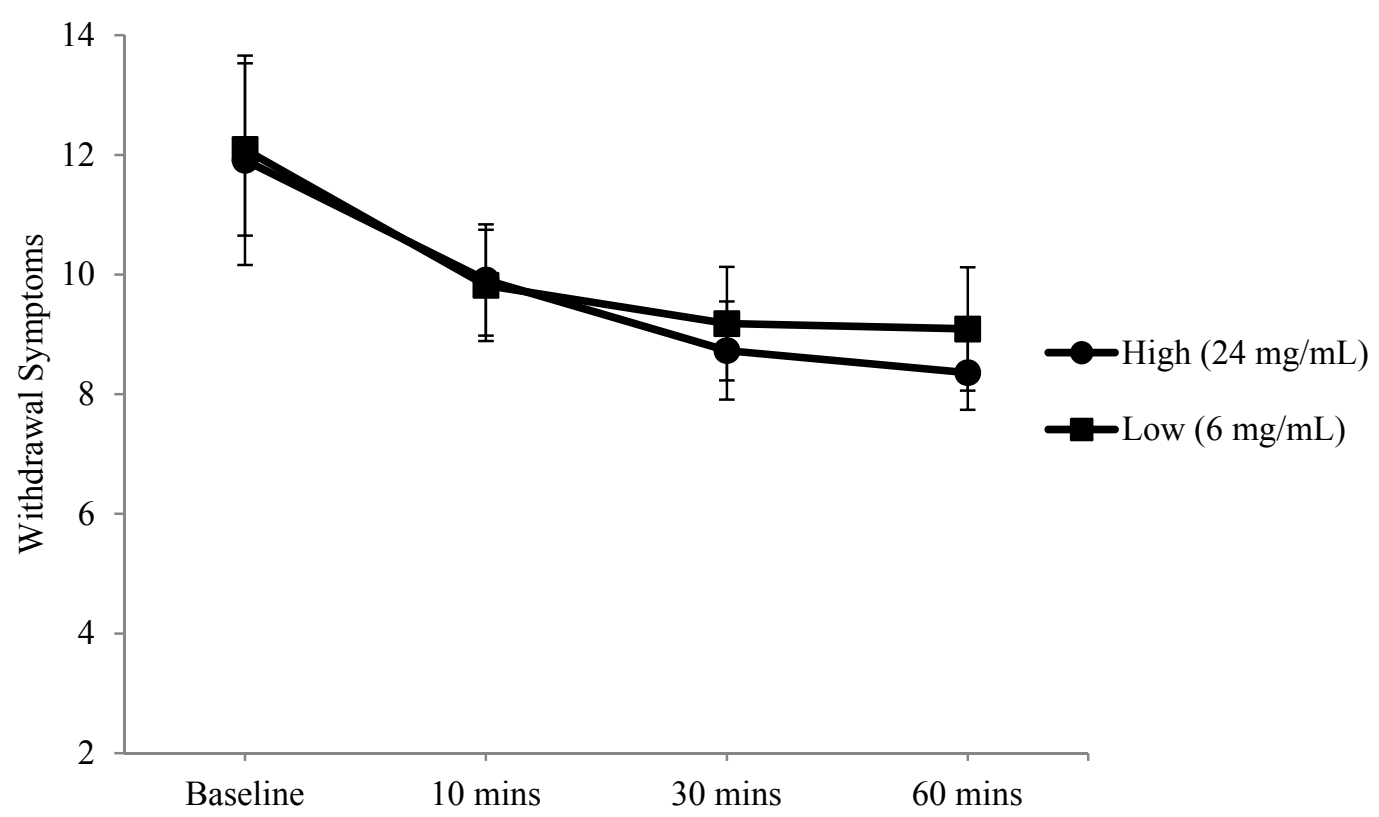

Fig. 3 Withdrawal symptoms vape at baseline, 10, 30 and $60 \mathrm{~min}$ for the high and low nicotine conditions $(N=11$; Error bars are \pm 1 SEM)

Mean (SD) percentage hit and satisfaction levels were 61.86 (31.50) and 60.70 (17.30) respectively in the high condition and 44.73 (23.00) and 46.89 (16.93) in the low condition. These differences did not reach statistical significance (hit: $Z=-1.60, p=0.11$; satisfaction: $Z=-1.69, p=0.09$ ).

Adverse effect reporting was generally low (data not shown) with throat irritation, mouth irritation, sweaty and light-headedness being most commonly reported (all under 20\%). One participant vomited $30 \mathrm{~min}$ into the first testing session (high nicotine condition). This participant had failed to consume breakfast and experienced anxiety over difficulty locating the study venue on a very hot day. Symptoms fully resolved within $20 \mathrm{~min}$ and the participant chose to continue with the study.

Overall mean adverse effect percentage ratings were slightly higher (by $2 \%)$ in the high: $10.91(8.60)$ versus low: 8.89 (5.65) nicotine condition (not statistically significant: $\mathrm{Z}=-1.17, \mathrm{P}=0.24$ ). Analysis of individual symptoms revealed no significant differences across conditions (all ps $>0.09$ ).

\section{DISCUSSION}

This study is the first to directly explore self-titration by allowing experienced e-cigarette users, habitually using high nicotine strength liquid, to vape ad libitum in the lab. The findings provide direct empirical evidence of a clear attempt to self-titrate; when given lower nicotine strength liquid, 
users increased their puff frequency and duration and consumed more liquid. As with tobacco smoking, compensation was partially effective; users reported equivalent reduction in urge to vape and withdrawal symptoms between conditions although blood nicotine levels were significantly higher in the high nicotine condition

Consumption of liquid was doubled in the low versus high nicotine condition; mean puff number increased from 48.36 to 70.73 and puff duration from 3.84 to $5.20 \mathrm{~s}$. These findings support the notion that users attempt to self-titrate by adjusting puffing behaviour to maximise nicotine delivery from a lower nicotine strength liquid. Indeed, indices of puffing patterns correlated positively with blood nicotine levels (with the exception of puff duration in the low nicotine condition). The phenomena of self-titration via compensatory puffing is well documented in the tobacco literature. Smokers increase puff frequency, duration and volume when switching to a lower nicotine cigarette whilst maintaining blood nicotine levels (Sutton et al., 1982). Similarly, here, although there was a 4-fold reduction in the nicotine strength of the low compared with the high liquid, corresponding plasma nicotine levels were only reduced by half at 30 and $60 \mathrm{~min}$, but were at the expected level at $10 \mathrm{~min}$ (i.e. approximately $1 / 4$ that of the high condition). Thus, compensatory puffing can be effective in raising blood nicotine levels but can take up to $30 \mathrm{~min}$ (at least with the concentrations used here) and, as with tobacco-smoking, does not appear to be complete (Ashton, Stepney \& Thompson, 1979; Russell, Wilson, Patel, Feyerabend \& Cole, 1975). Incomplete self-titration may be due to a 'saturation' effect, that is, a limit on the volume of liquid that an individual can comfortably consume within a given time period, or because a given level of compensatory puffing is sufficient to achieve subjective satisfaction and alleviation of craving and withdrawal symptoms.

Indeed, despite the difference in plasma nicotine concentrations, the magnitude of craving and withdrawal symptom reduction was similar across conditions suggesting effective compensatory puffing behaviour, at least in the short term. Hit and satisfaction were generally higher in the high nicotine condition but not significantly so. There was however, considerable individual variation and the lack of a statistically significant effect may be related to the small sample size. Effects of puffing topography associated with different nicotine strength liquids on subjective effects certainly merit further exploration. Adverse effects were uncommon and only $2 \%$ higher in the high nicotine condition.

Although our participants demonstrate clear evidence of compensatory puffing, self-titration was far from perfect with significantly higher plasma nicotine levels in the high nicotine condition. We observed a mean plasma nicotine boost of $33.77 \mathrm{ng} / \mathrm{mL}$ in $10 \mathrm{~min}$ with some individuals achieving 
considerably higher concentrations. This level exceeds those reported by other researchers using similar (Farsalinos et al., 2014; 2015) or higher nicotine e-liquid strength (Ramôa et al., 2015) and is equivalent to that observed in tobacco smokers (Russell, 1980, Russell et al., 1975) within 5 min of finishing a cigarette. The high plasma nicotine levels are likely due to a combination of: the device used, our ad libitum vaping design, $12 \mathrm{~h}$ nicotine abstinence requirement and the nature of our participants. The Aspire Nautilus tank used here is a more advanced system with more effective nicotine delivery to the aerosol (Farsalinos et al., 2016) compared to previous studies (Farsalinos et al., 2014; 2015; Ramôa et al., 2015). We also selected participants already familiar with using higher nicotine strength liquid and who had baseline salivary cotinine levels exceeding $100 \mathrm{ng} / \mathrm{mL}$. In fact, baseline salivary cotinine levels were very high (M: 467) similar to (indeed higher than) those typically seen in heavy smokers (334.83 ng/mL) (Russell, Jarvis, Devitt, Feyerabend, 1981). Although all participants were ex-smokers, a full smoking history and pre-quit salivary cotinine levels were not obtained so we cannot conclude whether nicotine exposure matched, exceeded or fell short of their pre-quit levels.

Our study is one of the first (Farsalinos et al., 2015) to use inbuilt EC software to capture information on puffing topography, a straightforward and arguably more ecologically valid procedure than CReSS pocket devices. However, the eVic does not capture puff flow rate and, as a safety feature, stops functioning after a $10 \mathrm{~s}$ puff/button press. Nevertheless, recent findings suggest that puff velocity does not influence nicotine yield (Talih et al., 2014), $>10 \mathrm{~s}$ puffs are rare (only $1.17 \%$ of all puffs in our study), and the CReSS device shares similar recording issues with a limit of 43 puffs. Despite methodological differences, puff durations under high and low nicotine conditions observed here are similar to those previously reported in experienced users using newer generation devices (Farsalinos et al., 2014; Hua et al., 2013) but longer than those reported for cartomiser (first-generation) devices (Behar, Hua, \& Talbot, 2015) and tobacco cigarettes (Hua et al., 2013). Our study was conducted in a controlled laboratory environment which may not reflect real-world puffing behaviour (Robinson, Hensel, Morabito \& Roundtree 2015) possibly over-estimating puffing. Indeed, the self-reported daily $\mathrm{mL}$ usually consumed (mean: $4.09 \pm 1.791$; see Table 1) supports this notion, although the $12 \mathrm{~h}$ abstinence period may have also contributed to the intense puffing patterns as participants strive to raise fallen nicotine levels. Similarly, the fixed device settings imposed here reflect standard second generation devices and allow a high degree of experimental control, but may not reflect how experienced third generation e-cigarette users behave.

To conclude, vapers may opt for, or switch to, lower nicotine strength liquid for a variety of reasons: the belief that it is healthier; weaning off nicotine; or due to the implementation of the EU Tobacco 
Products Directive (TPD). Our data suggest that experienced users tend to adjust their puffing patterns when switching to a lower nicotine liquid, maintaining adequate subjective effects, satisfaction and hit. It also suggests that very high nicotine levels are not absolutely necessary for alleviation of craving or withdrawal at least in acute conditions in the laboratory. Compensatory puffing patterns however, cannot fully compensate for the low nicotine delivery and increased puffing frequency results in increased costs and more frequent refills. Those currently using nicotine levels exceeding $20 \mathrm{mg} / \mathrm{mL}$ will be obliged to switch to lower nicotine strength liquids with the implementation of the TPD from May 2016. Our results suggest that such individuals will increase their liquid consumption. If health risk is related to dose, and longer, more frequent puffing is associated with higher temperatures, this is likely to increase risk of exposure to potential toxins and carcinogens (Kośmider et al., 2014) although exposure is still likely to be considerably lower than from cigarette smoke. Electronic cigarettes have seen an unprecedented evolution in technology over the last five to six years. Here, using $24 \mathrm{mg} / \mathrm{mL}$ nicotine strength liquid, we observed high blood nicotine levels achieved very quickly, matching and even exceeding those reported in tobacco smokers. Although it may be at the detriment of continued nicotine dependence, such effective nicotine delivery may be important to increase the appeal of e-cigarettes and augment smoking cessation rates.

\section{Declaration of competing interests}

We declare that we have received no support from any companies for this work and no nonfinancial conflicts of interest that would be considered relevant to this work. All authors have completed the conflict of interest Disclosure form at http://static.springer.com/sgw/documents/1378102/application/pdf/213_Conflict+of+Interest+formMar2012.pdf (available on request from the corresponding author).

LED has previously conducted research for several independent electronic cigarette companies. These companies had no input into the design, conduct or write up of the projects. She has also acted as a consultant for the pharmaceutical industry and as an expert witness in a patent infringement case.

$\mathrm{CK}$ and $\mathrm{OC}$ declare no competing interests.

MD and CF have previously supported other research groups by the quantification of cotinine, 3hydroxy cotinine and nicotine. These groups had no input into the design, conduct or write up of this project. 


\section{Details of contributors}

LED and OC conceived the original idea for this study and designed the study with input from CK and MD. CK, LED and OC managed the running of the participant facing experiment; OC and CK were responsible for the plasma extraction and $\mathrm{MD}$ and $\mathrm{CF}$ conducted the biochemical analysis of blood and saliva samples. CK and LED analysed the data and wrote the first draft with edits from OC, MD and CF. All authors had full access to all of the data and collectively take responsibility for the integrity of the data and accuracy of the data analysis. All authors read and approved the final version of the manuscript. Study guarantor: UEL.

\section{Role of the funding source}

The funders (UEL) had no involvement in the study design, data collection, analysis or interpretation of data or in the writing of the manuscript or the decision to submit the article for publication.

The authors had sole responsibility for the study design, collection, analysis and interpretation of data and for the writing of the manuscript and decision to submit for publication.

All authors had full access to all of the data (including statistical reports and tables) in the study and take responsibility for the integrity of the data and the accuracy of the data analysis

\section{Transparency declaration}

I, LED the lead author, affirm that the manuscript is an honest, accurate, and transparent account of the study being reported; no important aspects of the study have been omitted.

\section{Data sharing statement}

All participants gave informed consent for data sharing and full dataset will be available after publication of the manuscript on the UEL data repository.

\section{Acknowledgments}

This study forms part of a PhD research project and is funded by an 'Excellence Studentship' awarded to the second author from the University of East London (UEL). We also thank the participants for their time and participation in this research and the technical support staff at the School of Health, Sport and Bioscience: Mr Paul Boakes and Mr Rory Butterworth. 


\section{References}

ASH Action on Smoking and Health (2015). ASH Fact sheet on the use of electronic cigarettes (vapourisers) among adults in Great Britain. 2015-12-10.

URL:http://www.ash.org.uk/files/documents/ASH_891.pdf. Accessed: 2015-12-10.

(Archived by WebCite ${ }^{\circledR}$ at http://www.webcitation.org/6dgFwr9ne).

Ashton H, Stepney R, Thompson JW (1979). Self-titration by cigarette smokers. BMJ, 2(6186):357. PubMed PMID: 4917931.

Ashton, H., \& Watson, D. W. (1970). Puffing frequency and nicotine intake in cigarette smokers. Br Med J, 3(5724), 679-681.

Behar RZ, Hua M, Talbot P. (2015). Puffing Topography and Nicotine Intake of Electronic Cigarette Users. PLoS ONE, 10(2):1-18. PubMed PMID: 101319670.

Brown J, Beard E, Kotz D, Michie S, West R. (2014). Real-world effectiveness of e-cigarettes when used to aid smoking cessation: a cross-sectional population study. Addiction, 109(9):153140. PubMed PMID: 97445181.

Bullen C, McRobbie H, Thornley S, Glover M, Lin R, Laugesen M. (2010). Effect of an electronic nicotine delivery device (e cigarette) on desire to smoke and withdrawal, user preferences and nicotine delivery: randomised cross-over trial. Tob control, 19(2):98-103.

Dawkins L, Corcoran O. (2014). Acute electronic cigarette use: nicotine delivery and subjective effects in regular users. Psychopharmacology, 231(2):401-7.

Eissenberg T. (2010). Electronic nicotine delivery devices: ineffective nicotine delivery and craving suppression after acute administration. Tob control, 19(1):87-8.

Etter J-F. (2015). Explaining the effects of electronic cigarettes on craving for tobacco in recent quitters. Drug Alcohol Depend, 148:102-108, ISSN 0376-8716, http://dx.doi.org/10.1016/j.drugalcdep.2014.12.030 (0).

Etter, J-F., Le Houezec, J. \& Perneger, TV. (2003). A self-administered questionnaire to measure dependence on cigarettes: The Cigarette Dependence Scale. Neuropsychopharm, 28: 359370.

Fagerström K. (2012). Determinants of tobacco use and renaming the FTND to the Fagerström Test for Cigarette Dependence. Nicotine Tob Res, 14(1):75-8. PubMed PMID: 2011-30003-008.

Farsalinos, K. E., Romagna, G., Tsiapras, D., Kyrzopoulos, S., \& Voudris, V. (2013). Evaluating nicotine levels selection and patterns of electronic cigarette use in a group of" vapers" who had achieved complete substitution of smoking. Substance abuse: research and treatment, 7, 139 .

Farsalinos KE, Spyrou A, Stefopoulos C, Tsimopoulou K, Kourkoveli P, Tsiapras D, et al. (2015). Nicotine absorption from electronic cigarette use: Comparison between experienced consumers (vapers) and naïve users (smokers). Sci Rep, 5, 11269; doi: 10.1038/srep11269.

Farsalinos KE, Spyrou A, Tsimopoulou K, Stefopoulos C, Romagna G, Voudris V. (2014). Nicotine absorption from electronic cigarette use: comparison between first and newgeneration devices. Sci Rep, 2014;4 (4133). doi:10.1038/srep04133

Farsalinos, KE., Yannovits, N., Sarri, R., Voudris, V. \& Poulas, K. (2016). Protocol proposal for, and evaluation of, consistency in nicotine delivery from the liquid to the aerosol of electronic cigarettes atomizers: regulatory implications. Addiction, online ahead of print. Doi: $10.1111 /$ add.13299.

Hua M, Yip H, Talbot P. (2013). Mining data on usage of electronic nicotine delivery systems (ENDS) from YouTube videos. Tob Control, 22(2):103-6. PubMed PMID: 2011945261.

Kośmider L, Sobczak A, Fik M, Knysak J, Zaciera M, Kurek J, Goniewicz ML. (2014). Carbonyl compounds in electronic cigarette vapors - effects of nicotine solvent and battery output voltage. Nicotine Tob Res, 16(10): 1319-1326.

Lee YH, Gawron M, Goniewicz ML. (2015). Changes in puffing behavior among smokers who switched from tobacco to electronic cigarettes. Addict Behav, 9;48(0):1-4. 
Polosa, R., Caponnetto, P., Cibella, F., \& Le-Houezec, J. (2015). Quit and smoking reduction rates in vape shop consumers: a prospective 12-month survey. International journal of environmental research and public health, 12(4), 3428-3438.

Polosa R, Morjaria JB, Caponnetto P, Campagna D, Russo C, Alamo A, et al. (2014). Effectiveness and tolerability of electronic cigarette in real-life: A 24-month prospective observational study. Intern Emerg Med, 9(5):537-46.

Ramôa CP, Hiler MM, Spindle TR, Lopez AA, Karaoghlanian N, Lipato T, et al. (2015). Electronic cigarette nicotine delivery can exceed that of combustible cigarettes: a preliminary report. Tob Control.

Robinson RJ, Hensel EC, Morabito PN, Roundtree KA. (2015). Electronic Cigarette Topography in the Natural Environment. PLoS ONE, 10(6):1-14. PubMed PMID: 103565825.

Russell, MAH. (1980) Nicotine intake and its regulation. J Psychosom Res, 24(5):253-64.

Russell MAH, Jarvis MJ, Devitt G, Feyerabend C. (1981). Nicotine intake by snuff users. $B M J$, 283(6295):814-7.

Russell MAH, Sutton SR, Iyer R, Feyerabend C, Vesey CJ. (1982). Long-Term Switching to LowTar Low-Nicotine Cigarettes. Br J Addict, 77(2):145-58. PubMed PMID: 6272769.

Russell MA, Wilson C, Patel UA, Feyerabend C, Cole PV. (1975). Plasma nicotine levels after smoking cigarettes with high, medium, and low nicotine yields. BMJ, 2(5968):414-6. PubMed PMID: PMC1681802.

Sutton SR, Russell MAH, Iyer R, Feyerabend C, Saloojee Y. (1982). Relationship between cigarette yields, puffing patterns, and smoke intake: Evidence for tar compensation? BMJ, 285(6342):600-3.

Talih S, Balhas Z, Eissenberg T, Salman R, Karaoghlanian N, El Hellani A, et al. (2014) Effects of User Puff Topography, Device Voltage, and Liquid Nicotine Concentration on Electronic Cigarette Nicotine Yield: Measurements and Model Predictions. Nicotine Tob Res, Sep 3. PubMed PMID: 25187061.

Trtchounian A, Williams M, Talbot P. (2010). Conventional and electronic cigarettes (e-cigarettes) have different smoking characteristics. Nicotine Tob Res, 12(9):905-12. PubMed PMID: 53376549.

West R, Hajek P. (2004). Evaluation of the mood and physical symptoms scale (MPSS) to assess cigarette withdrawal. Psychopharmacology, 177(1/2):195-9. PubMed PMID: 15244922.

Woodward M, \& Tunstall-Pedoe, HU. (1993). Self-titration of nicotine: evidence from the Scottish Heart Health Study. Addiction, 88(6), 821-830. 